\title{
Hipertensión intracraneal traumática refractaria: el papel de la craniectomía descompresiva
}

\author{
Refractory traumatic intracranial hypertension: the role of decompressive craniectomy
}

Luis R. Moscote-Salazar ${ }^{1,2 *}$, Hernando R.I Alvis-Miranda², Yancarlos Ramos-Villegas², Loraine QuintanaPajaro², Andrés M. Rubiano ${ }^{3}$, Gabriel Alcalá-Cerra4, Juan B. González-Torres ${ }^{5}$ y Alexis R. Narváez-Rojas 2,6 ${ }^{1}$ Departamento de Neurocirugía, Universidad de Cartagena, Colombia; ${ }^{2}$ Cartagena Neurotrauma Research Group, Universidad de Cartagena, Colombia; ${ }^{3}$ Cuidados Neurocríticos, Fundación MEDITECH, Colombia; ${ }^{4}$ Departamento de Neurocirugía, Universidad de Cartagena, Colombia; ${ }^{5}$ Departamento de Neurocirugía Pediátrica, Hospital Infantil Manuel de Jesús Rivera, La Mascota, Managua, Nicaragua; ${ }^{6}$ Facultad de Ciencias Médicas, Universidad Nacional Autónoma de Nicaragua, Managua, Nicaragua

\section{Resumen}

El trauma craneoencefálico, según la Organización Mundial de la salud, se estima que para el año 2020 será la tercera causa de morbimortalidad en el mundo. La hipertensión intracraneal refractaria al manejo médico es la causante de la mayor mortalidad en esta población de pacientes. Existen diversas medidas para el control de la hipertensión intracraneal, entre ellas las quirúrgicas. La craniectomía descompresiva ha sido utilizada sistemáticamente para tratar la hipertensión intracraneal secundaria al infarto cerebral, la hemorragia subaracnoidea, la hemorragia intracerebral y el trauma. Se hace una revisión de la literatura para describir los mecanismos fisiopatológicos de la lesión cerebral traumática, así como también los tipos y las indicaciones de este procedimiento.

PALABRAS CLAVE: Craniectomía. Craniectomía descompresiva. Hipertensión intracraneal. Neurotrauma.

\begin{abstract}
Traumatic brain injury according to the World Health Organization estimates that by 2020 will be the third leading cause of morbidity and mortality worldwide. Intracranial hypertension refractory to medical management is the cause of increased mortality in neurotrauma. There are various measures to control intracranial hypertension, including surgical. Decompressive craniectomy has been routinely used to treat intracranial hypertension secondary to cerebral infarction, subarachnoid hemorrhage, intracerebral hemorrhage and trauma. We review the literature to describe the mechanisms, types and indications for this procedure.
\end{abstract}

KEY WORDS: Craniectomy. Decompressive craniectomy. Intracranial hypertension. Neurotrauma.

\section{Correspondencia:}

*Luis Rafael Moscote-Salazar 


\section{Introducción}

La Organización Mundial de la Salud estima que, para el año 2020, el trauma craneoencefálico (TCE) será la tercera causa de morbimortalidad en todo el mundo ${ }^{1}$. Anualmente, en los EE.UU. se producen cerca de 1.7 millones de visitas en los servicios de urgencias por este motivo de consulta, lo que conlleva más de 275,000 hospitalizaciones y 52,000 fallecimientos $^{2}$. En países como Colombia, donde la principal causa de muerte es la violencia, entre el 49 y el $70 \%$ corresponden a TCE. A pesar de que en las estadísticas nacionales no exista una especificación para cada causa de TCE, se ha estimado su incidencia por medio del estudio de las lesiones en accidentes de tránsito, que a su vez proveen una gran cantidad de $\operatorname{casos}^{3,4}$. Para finales del año 2010, el Instituto Nacional de Medicina Legal y Ciencias Forenses de dicho país había revelado una cifra total de 45,022 víctimas de accidentes de tránsito, de las cuales $4471(9.9 \%)$ sufrieron lesiones en la cabeza, que fue el tercer lugar más afectado después del politrauma (lesiones en más de una región anatómica aparte de la cabeza) y del trauma de las extremidades ${ }^{4}$. De estos casos, 1535 (34.3\%) correspondieron a lesiones fatales y $2936(65.7 \%)$ a lesiones no fatales, con lo cual se evidencia la alta tasa de mortalidad que genera el TCE secundario a esta causa ${ }^{4}$.

Con el pasar del tiempo, el manejo médico-quirúrgico del TCE ha variado sustancialmente. En un principio, todos los pacientes eran tratados fuera de la unidad de cuidados intensivos sin emplear ningún método de neuromonitore $0^{5}$, lo que resultaba en cifras de mortalidad por encima del $60 \%$. A partir de la década de 1970, con la atención de estos enfermos en la unidad de cuidados intensivos y la introducción de nuevas técnicas de monitoreo, incluida la de la presión intracraneal (PIC), se logró conocer en tiempo real cada uno de los eventos fisiopatológicos que trascurren durante su evolución, y se pudo tratarlos de manera más racional que con el manejo anterior, dando lugar a lo que hoy en día se conoce como neurointensivismo del trauma craneal. Este ha logrado disminuir la mortalidad a cifras que oscilan de un 20 a un $45 \%^{3}$.

\section{Hipertensión intracraneal}

La PIC elevada es la principal causa de muerte en los pacientes con lesión traumática cerebral y contribuye a la lesión cerebral secundaria si no es correctamente manejada ${ }^{6}$. La teoría de Monro-Kellie propone que el cráneo rígido está ocupado por tres volúmenes, que son sangre, cerebro y líquido cefalorraquídeo, y cualquier volumen adicional, como hematomas, edema cerebral o hidrocefalia, resultará en un aumento de la PIC cuando los desplazamientos compensatorios de los volúmenes primarios sean excedidos$^{7}$. La capacidad de almacenar hasta $150 \mathrm{cc}$ del nuevo volumen intracraneal sin un significativo incremento en la PIC ocurre por desplazamiento de sangre venosa hacia la circulación general; el desplazamiento hacia fuera del líquido cefalorraquídeo depende del tiempo y de la edad del paciente ${ }^{7}$. Las personas mayores tienden a presentar más atrofia cerebral y de esta manera reacomodan mayor cantidad de volumen, que lentamente se expande ${ }^{8}$. Las personas jóvenes con procesos agudos, en su contraparte, se convierten en sintomáticos más rápido ante los mismos procesos fisiopatológicos ${ }^{9}$. Las lesiones ocupantes de espacio serán discutidas en la sección subsecuente y se asume que estas lesiones han sido evacuadas quirúrgicamente. La autorregulación cerebral anormal, el flujo sanguíneo y el edema cerebral persisten como causa de elevación de la $\mathrm{PIC}^{7}$.

Se ha demostrado en estudios clínicos que los pacientes con trauma craneal y PIC mayor de $20 \mathrm{mmHg}$, en particular cuando son refractarios al tratamiento, tienen un peor pronóstico clínico y son más propensos a presentar síndromes de herniación cerebral. Existe también evidencia reciente de que la presión de perfusión cerebral por debajo de $60-70 \mathrm{mmHg}$ se asocia con una disminución de la oxigenación del parénquima cerebral, alteración del metabolismo y peor pronóstico ${ }^{10}$. El objetivo del neuromonitoreo y del tratamiento es por lo menos mantener una perfusión cerebral adecuada, la oxigenación y el metabolismo, y además limitar la progresión de la PIC elevada, los fenómenos de desaturación y el edema, entre otros ${ }^{11}$.

Cualquier incremento de volumen adicional por hiperemia o por la presencia de hematomas disminuirá el volumen de los otros componentes y conllevará un incremento de la PIC (Fig. 1) . $^{7}$ Cerca de un tercio de los pacientes con lesión craneal difusa y la mitad de aquellos que presentan masas intracraneales tienen la PIC elevada ${ }^{12}$. La PIC típica del adulto varía en un rango de 5 a $15 \mathrm{mmHg}$, y los valores pediátricos entre 0 y $10 \mathrm{mmHg}^{13}$. La elevación persistente de la PIC se ha asociado con un pobre pronóstico y la mortalidad se relaciona directamente con el grado y la duración 


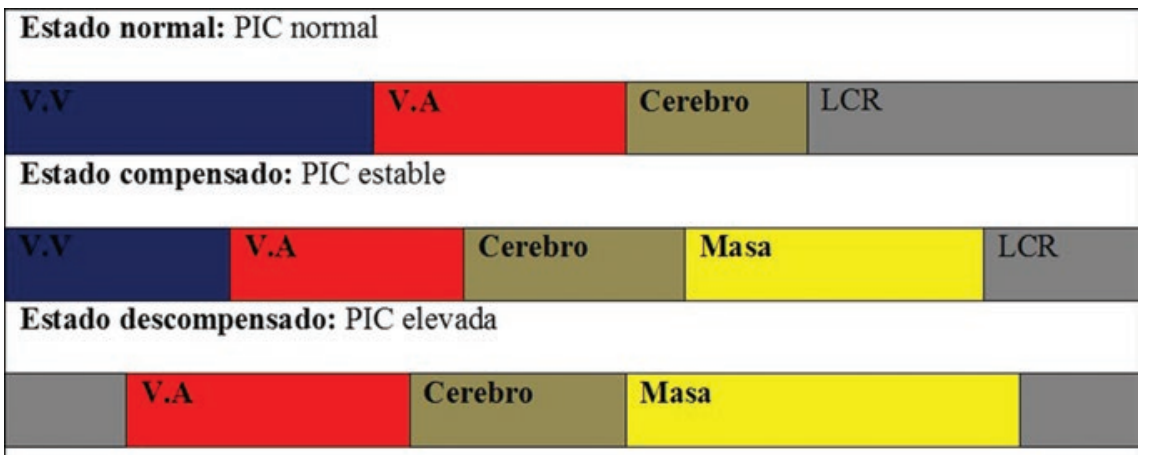

Figura 1. Descripción de la teoría de Monroe-Kelly. LCR: líquido cefalorraquídeo; PIC: presión intracraneal; VA: volumen arterial; VV: volumen venoso.

de la PIC elevada ${ }^{14}$. Cuando la PIC aumenta, la presión de perfusión cerebral generalmente disminuye ${ }^{15}$.

\section{Síndromes de herniación}

Los cuatro tipos de herniación más frecuentes son la subfalcina, la transtentorial (uncal), la axial (central) y la amigdalina; cada una implica diferentes estructuras y se asocia con síndromes clínicos específicos. Cualquier masa intracraneal expansiva puede resultar en el incremento de la PIC y una herniación del tejido cerebral a través de las aperturas durales y calvarias ${ }^{16}$. La herniación subfalcina 0 cingulada usualmente resulta de una masa hemisférica expansiva en la fosa anterior y media, la cual causa que el giro cingulado se hernie por debajo del borde libre de la hoz del cerebro ${ }^{16}$. Dependiendo del grado de herniación, una o ambas arterias pericallosas pueden estar comprimidas, causando paraparesia. La herniación transtentorial o uncal típicamente resulta de una masa expansiva en la fosa media o temporal ${ }^{17}$. En la hernia del uncus, esta estructura se hernia a través del borde libre de la tienda del cerebelo, resultando en la compresión del tercer nervio craneal homolateral y el pedúnculo cerebral, con hemiparesia contralateral ${ }^{16}$. La herniación uncal también puede producir compresión de la arteria cerebral posterior y dar lugar a infarto o isquemia occipital. Las herniaciones central y amigdalina pueden resultar de un incremento generalizado de la PIC o de la presencia de masas supratentoriales; esta se manifiesta como disminución del estado de conciencia, irregularidades respiratorias, hipertensión y bradicardia ${ }^{16}$. La herniación amigdalina también puede estar asociada con masas de la fosa posterior y resulta en un desplazamiento de las amígdalas cerebelosas a través del foramen magno, comprimiendo la médula oblonga y llevando finalmente a depresión respiratoria ${ }^{18}$. Aunque estos son síndromes clínicos bien definidos asociados a ciertas patologías, es necesario disponer de una tomografía computarizada (TC) cerebral simple ${ }^{19}$.

\section{Hipertensión intracraneal y craniectomía descompresiva}

Diversos autores han hecho modificaciones quirúrgicas para el manejo de la hipertensión endocraneal refractaria, y así se han implementado muchos tipos de craniectomía descompresiva. Las variaciones de los resultados en los estudios pueden explicarse por los diferentes tipos de técnicas quirúrgicas existentes ${ }^{20}$. En este momento, la craniectomía frontotemporoparietal/ hemisferio craniectomía por el edema confinado a uno de los hemisferios cerebrales y la craniectomía bifrontal por el edema difuso son las intervenciones recomendadas para corregir la hipertensión intracraneal refractaria ${ }^{21}$. Las mejoras en las técnicas de craniectomía descompresiva, apoyadas en una gran descompresión, se han implantado para aumentar el efecto descompresivo de la técnica, evitar las complicaciones quirúrgicas y propiciar su buen manejo y evolución ${ }^{21}$. No obstante, estos métodos son tentativamente experimentales, pero en la mayoría de los reportes y las reducidas poblaciones de estudio estas experiencias son valiosas. En la actualidad no existen pruebas definitivas respecto a la eficacia de la craniectomía descompresiva en el tratamiento del TCE ${ }^{22}$.

\section{Los dos tipos de craniectomías: hemicraniectomía y craniectomía bilateral}

Al realizar el plan quirúrgico hay algunos factores para tener en cuenta: la ubicación, el hemisferio, el tamaño de la descompresión, la técnica de la duramadre, el colgajo óseo, etc. La TC nos determinará 
la posición (frontal, temporal, parietal u occipital) y el hemisferio (unilateral o bilateral) ${ }^{22}$. Algunas indicaciones para la hemicraniectomía descompresiva son las lesiones unilaterales, tales como el edema unilateral, las contusiones, la hemorragia extradural o subdural, y la desviación de la línea media; por otro lado, la descompresión bifrontal se utiliza generalmente para el edema cerebral difuso, sin cambio de la línea media $^{23}$.

Una vez adoptada la decisión, debe tratarse al máximo de hacer la eliminación de hueso lo más grande posible. Se ha recomendado que el tamaño de la descompresión debe ser, como mínimo, de $14 \mathrm{~cm}$ (anteroposterior) por $12 \mathrm{~cm}$ (superoinferior) si la intención es realizar una craniectomía frontotemporoparietal ${ }^{22}$.

La técnica ideal implica la eliminación de hueso en todo el hemicráneo. Los reparos anatómicos son la raíz del hueso malar (que permite la identificación del suelo de la fosa temporal), el asterion (confluencia de las suturas lambdoidea, occipitomastoidea y escamosas, que indica la zona de transición entre los senos transverso y sigmoideo), el punto anatómico ubicado por detrás de la sutura frontocigomática y por debajo del comienzo de la línea temporal superior (identifica el pterion e indica la ubicación de los lóbulos frontal, temporal y orbitaria cavidades), el inion, la glabela y la línea media (delinea el curso del seno sagital superior) ${ }^{21-23}$.

\section{Incisiones cutáneas para la hemicraniectomía descompresiva}

Debido a que el objetivo es la exposición de todo el hemicráneo, deben realizarse dos tipos de incisiones: incisión grande en signo de interrogación invertido frontotemporoparietal o incisión tipo L.G. Kempe modificada, o incisión de barra en $T^{21,22}$.

\section{Incisión grande en signo de interrogación invertido frontotemporoparietal}

Esta incisión es rápida y fácil, pero tiene riesgo de isquemia y de dehiscencia de la herida. Se inicia en la región pretemporal, continúa posteriormente a lo largo de la línea media de la inion, luego gira de forma brusca al oído y paralela a una línea que se extiende desde el inion hasta la raíz del hueso malar ${ }^{24}$. La incisión debe bordear las partes superior y anterior de la oreja lo más cerca posible y ampliar $1 \mathrm{~cm}$ por debajo de la raíz del hueso malar. Con esta incisión, el hemicráneo se expone en la línea media, a lo largo de la línea del seno transverso. También permite una gran exposición de la fosa temporal. Hay que intentar preservar la arteria temporal superficial, que asegura un buen flujo de sangre al colgajo de piel. Ahora, sobre el periostio puede practicarse una incisión con el uso de electrocauterio, y a continuación puede ser replegado el colgajo cutáneo ${ }^{22}$.

\section{Incisión tipo L.G. Kempe modificada o incisión en barra en $T$}

Con esta incisión hay un mejor flujo de sangre a los colgajos de piel, y por lo tanto un menor riesgo de dehiscencia, en especial en la parte posterior de la incisión. La debilidad de la incisión ya se ha descrito previamente. En la piel cabelluda se realiza una incisión en la línea media desde la inserción de la piel cabelluda hasta el inion, y después de esto se crea una extremidad a la incisión, que forma la «barra en T» de 1-2 cm anterior al trago, y que se extiende superiormente, $1 \mathrm{~cm}$ detrás de la sutura coronal, hasta encontrar la incisión sagital en la línea media ${ }^{22,24}$.

\section{Colgajo óseo para la hemicraniectomía descompresiva}

A nivel superior a la raíz del hueso zigomático se realiza un solo agujero de trépano, y de esta manera se delinea el suelo de la fosa temporal. El asterion debe exponerse al replegar el tejido hacia caudal, suavemente; realizando esta maniobra pueden visualizarse muy bien anatómicamente las porciones inferiores de los lóbulos temporal y occipital. Para evitar el seno transverso es necesario estar al menos $1 \mathrm{~cm}$ rostral al asterion. La sutura lambdoidea se cruza cuando el colgajo óseo se extiende posteriormente, y a continuación, la broca de perforación se gira en paralelo a $Y, 1 \mathrm{~cm}$ medial a la sutura lambdoidea, hasta llegar a un punto de $1 \mathrm{~cm}$ de la línea media $^{24,25}$.

Se hace el corte paralelo al seno sagital, atravesando de nuevo la sutura. La perforación continúa hacia la barra supraorbitaria y la craneotomía se continúa hacia delante incluyendo el suelo de la fosa frontal en la mayor medida posible; hay que mantenerse lo más cerca posible del borde orbitario. Se sigue haciendo el corte posterolateralmente hacia la sutura frontocigomática y lo más cerca posible del pterion. En este punto, la cuchilla de corte se retira y se vuelve a 
insertar en el orificio de fresa en la raíz del hueso malar $^{22,25}$.

La segunda línea de corte se crea incluyendo el suelo de la fosa temporal y se extiende tan lejos como sea posible hacia la parte anterior de la punta temporal. El colgajo óseo se retira haciendo palanca con el pterion como punto de apoyo ${ }^{22}$.

\section{Apertura dural}

Para este paso pueden utilizarse tres formas diferentes de abrir la duramadre: en forma de estrella, en forma de $\mathrm{C}$ o con una incisión en cruz. La incisión en forma de $C$ es una de las más utilizadas para la apertura de la duramadre, que va desde la punta temporal del lóbulo temporal y se curva de nuevo cerca de $8 \mathrm{~cm}$ cruzando la cisura de Silvio, y termina en la región frontal; de esta manera, los hematomas subyacentes pueden ser evacuados. Una vez que se garantiza la hemostasia, la duramadre puede colocarse de nuevo sobre la superficie del cerebro, y se coloca un fragmento grande de sustituto dural sobre la duramadre ${ }^{25}$.

\section{Sustitutos durales y selladores}

En el cierre de la duramadre pueden utilizarse esponjas de gelatina absorbible $\left(\right.$ Gelfoam $^{\circledR}$, Pharmacia \& Upjohn, Kalamazoo, Michigan, EE.UU.), sustitutos durales y selladores durales. Los sustitutos de duramadre están diseñados para ser colocados como un injerto laminar sobre defectos durales o suturado en su lugar ${ }^{26}$. Estos pueden ser tejidos autólogos, tales como pericráneo o fascia lata, o sustitutos durales artificiales derivados principalmente de tendón bovino (DuraGen $^{\circledR}$, DuraGen Plus ${ }^{\circledR}$, Integra Life Sciences, Plainsboro, New Jersey, EE.UU.; DuraMatrix ${ }^{\circledR}$, Stryker, Cambridge, Massachusetts, EE.UU.; TissuDura ${ }^{\circledR}$, Baxter Healthcare SA, Zúrich, Suiza), y otros derivados de piel fetal bovina ${ }^{26}$.

Con el objetivo de reforzar el reparado principalmente de la duramadre o como adyuvantes de los sustitutos durales pueden utilizarse los selladores durales, como DuraSeal ${ }^{\circledR}$ (polietileno glicol de hidrogel, Confluente Surgical Inc., Waltham, Massachusetts, EE.UU.), Bioglue $^{\circledast}$ (glutaraldehído, albúmina bovina, Cryolife, Kennesaw, Georgia, EE.UU.), Tissucol ${ }^{\circledR}$ (fibrinógeno humano, trombina, albúmina y aprotinina), Tissee $^{\circledR}$ (fibrinógeno humano, trombina y aprotinina, Baxter International Inc., Westlake Village, California, EE.UU.), Evicel $^{\circledR}$ (fibrinógeno y trombina humanos, Johnson \&
Johnson Wound Management, Ethicon Inc., SomerviIle, New Jersey, EE.UU.) ${ }^{21}$ y Seprafilm ${ }^{\circledR}$ (celulosa hialuronato/carboximetil) ${ }^{27}$.

\section{Craniectomía descompresiva bilateral}

\section{Cierre de la piel}

La piel se cierra sobre la esponja de gelatina absorbible o el sustituto dural o sellador utilizando $\mathrm{Vi-}$ cryl ${ }^{\circledR}$ 2-0 (Ethicon, Johnson \& Johnson Professionals, Inc., Somerville, New Jersey, EE.UU.), y típicamente

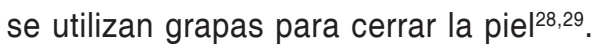

\section{Gestión del colgajo óseo en la craniectomía}

El colgajo óseo en la craniectomía puede descartarse, ser insertado en un bolsillo subcutáneo abdominal en el cuadrante inferior izquierdo o conservado en un banco de huesos ${ }^{21,30,31}$. Las consecuencias de descartar el colgajo óseo son obvias, pues requiere una craneoplastia con reconstrucción intraoperatoria, haciendo más costoso el procedimiento y sacrificando la estética. En algunos centros prefieren desprenderse de él y se apoya el uso de implantes protésicos de metacrilato de metilo, sobre todo porque más de la mitad de los pacientes con lesión grave del sistema nervioso central tienen procesos infecciosos sistémicos de algún tipo ${ }^{21}$. Cuando el colgajo óseo se conserva en el interior del cuerpo, por lo general se remodelan los bordes del hueso en algún grado. Por lo tanto, mantener el hueso congelado en un banco de huesos es una opción sin riesgos de remodelación ósea y ofrece excelentes resultados cosméticos ${ }^{32}$. Cuando el injerto óseo autógeno no está disponible para la craneoplastia pueden utilizarse materiales sintéticos, tales como tantalio, Silastic ${ }^{\circledR}$, titanio, acrílico prefabricado, sustituto sintético del hueso u otro material similar fabricado para su implantación en el cuerpo ${ }^{31}$.

\section{Efectos de una mala técnica}

Como se ha indicado anteriormente, la Craniectomia Descompresiva $(C D)$ es un procedimiento quirúrgico que reduce el daño secundario debido a un aumento incontrolado de la PIC, pero no corrige la lesión primaria $^{33}$. La aplicación de técnicas inapropiadas para CD, tales como no suavizar los bordes óseos, no hacer la extracción de hueso lo más grande posible, realizar 
aproximaciones equivocadas como única descompresión subtemporal o solo descompresión frontotemporal, puede generar lesiones cerebrales iatrogénicas e incluso herniación cerebral a través de la craniectomía ${ }^{34}$. No ser fiel a la técnica descrita puede tener resultados catastróficos y, en el peor resultado posible, llevar a la muerte del paciente. Los resultados inconsistentes y las opiniones en conflicto sobre la CD pueden deberse a la variación sustancial de su empleo ${ }^{21}$.

\section{Evidencia del uso de la craniectomía descompresiva}

Existen cuatro ensayos clínicos controlados publicados hasta la fecha ${ }^{35-38}$. De ellos, el más reciente, publicado en 2016, incluyó 23 países y una muestra de 408 pacientes, y demostró que el uso de la craniectomía descompresiva en la hipertensión intracraneal refractaria disminuye la mortalidad, pero aumenta el estado vegetativo, por lo cual se recomienda valorar con cada paciente y sus familiares la utilización de la técnica ${ }^{38}$.

\section{Conclusión}

La correcta mecánica del neurotrauma está orientada a ampliar el efecto de descompresión, prescindiendo de las complicaciones quirúrgicas, y de esta forma facilitar su eventual resolución. La aplicación de técnicas de descompresión inapropiadas puede representar la muerte para el paciente. Aún hay opiniones encontradas debido a la variación en la aplicación de la técnica sustentada en este artículo. El TCE es en un problema de salud pública, ya que representa muchas veces incapacidad en las personas que lo padecen por la alta morbilidad y la mortalidad que a este le atañe, debido a las lesiones que genera. La craniectomía descompresiva es una estrategia neuroquirúrgica práctica que permite disminuir la PIC cuando han fallado las medidas de primera línea para disminuirla.

\section{Conflicto de intereses}

Los autores declaran no tener ningún conflicto de intereses.

\section{Responsabilidades éticas}

Protección de personas y animales. Los autores declaran que para esta investigación no se han realizado experimentos en seres humanos ni en animales.
Confidencialidad de los datos. Los autores declaran que han seguido los protocolos de su centro de trabajo sobre la publicación de datos de pacientes.

Derecho a la privacidad y consentimiento informado. Los autores declaran que en este artículo no aparecen datos de pacientes.

\section{Bibliografía}

1. Murray CJ, Lopez AD. Alternative projections of mortality and disability by cause 1990-2020: Global Burden of Disease Study. Lancet. 1997;349:1498-504.

2. Centers for Disease Control and Prevention. Report to Congress on Traumatic Brain Injury in the United States: Epidemiology and Rehabilitation. National Center for Injury Prevention and Control; Division of Unintentional Injury Prevention. Atlanta, GA. 2015. Disponible en: https:// www.cdc.gov/traumaticbraininjury/pdf/TBI_Report_to_Congress_Epi_ and Rehab-a.pdf

3. Honeybul S. Decompressive craniectomy for severe traumatic brain injury: a review of its current status. J Neurol Neurophysiol S9. 2012. Disponible en: https://www.omicsonline.org/decompressive-craniectomy-for-severe-traumatic-brain-injury-a-review-of-its-current-status-2155-9562.S9-001.php?aid=5204

4. Moreno Lozada SL. Comportamiento de muertes y lesiones accidentales. Colombia, 2013. Bogota; 2013. Disponible en: http://www.medicinalegal. gov.co/documents/20143/49517/Muertes+y+lesiones+accidentales.pdf. Ultimo acceso: 10 de marzo de 2018

5. Guidelines for minor head injured patients' management in adult age. The Study Group on Head Injury of the Italian Society for Neurosurgery. J Neurosurg Sci. 1996;40:11-5.

6. Coronado VG, McGuire LC, Sarmiento K, Bell J, Lionbarger MR, Jones $C D$, et al. Trends in traumatic brain injury in the U.S. and the public health response: 1995-2009. J Safety Res. 2012;43:299-307.

7. Mokri B. The Monro-Kellie hypothesis: applications in CSF volume depletion. Neurology. 2001;56:1746-8.

8. Rangel-Castilla L, Rangel-Castillo L, Gopinath S, Robertson CS. Management of intracranial hypertension. Neurol Clin. 2008;26:521-41.

9. Rohlwink UK, Zwane E, Graham Fieggen A, Argent AC, Le Roux PD, Figaji AA. The relationship between intracranial pressure and brain oxygenation in children with severe traumatic brain injury. Neurosurgery. 2012;70:1220-31.

10. Nekludov M, Bellander B-M, Mure M. Oxygenation and cerebral perfusion pressure improved in the prone position. Acta Anaesthesiol Scand. 2006;50:932-6.

11. Egea-Guerrero JJ, Gordillo-Escobar E, Revuelto-Rey J, Enamorado-Enamorado J, Vilches-Arenas A, Pacheco-Sánchez M, et al. Clinical variables and neuromonitoring information (intracranial pressure and brain tissue oxygenation) as predictors of brain-death development after severe traumatic brain injury. Transplant Proc. 2012;44:2050-2.

12. Maghool F, Khaksari M, Siahposht Khachki A. Differences in brain edema and intracranial pressure following traumatic brain injury across the estrous cycle: involvement of female sex steroid hormones. Brain Res. 2013;1497:61-72.

13. Abdalla Mohamed A, Ahmed Ibrahim W, Fayez Safan T. Hemodynamic and intracranial pressure changes in children with severe traumatic brain injury. Egypt J Anaesth. 2011;27:273-8.

14. Geeraerts T, Menon DK. Le monitorage de la pression intracrânienne améliore-t-il le devenir des traumatisés crâniens graves? Ann Fr Anesth Reanim. 2010;29:e171-5.

15. Cianfoni A, Cha S, Bradley WG, Dillon WP, Wintermark M. Quantitative measurement of blood-brain barrier permeability using perfusion-CT in extra-axial brain tumors. J Neuroradiol. 2006;33:164-8.

16. Kan PKY, Chu MHM, Koo EGY, Chan MTV. Brain herniation. En: Complications in Neuroanesthesia. London: Academic Press - Elsevier; 2016. p. 3-13.

17. Hou K, Zhu X, Zhao J, Zhang Y, Gao X, Jiang K, et al. Efficacy of reinforced restriction of physical activity on chronic subdural hematoma: prospective pilot study. World Neurosurg. 2018;110:e1011-6.

18. Patil AA, Hahn FJ, Leibrock LG. Axial herniation with brain stem deformity as a cause of sleep apnea. Clin Neurol Neurosurg. 1991;93:65-7.

19. Nguyen JP, Djindjian $M$, Brugières $P$, Badiane $S$, Melon $E$, Poirier J. Anatomy-computerized tomography correlations in transtentorial brain herniation. J Neuroradiol. 1989;16:181-96.

20. Timofeev I, Czosnyka M, Nortje J, Smielewski P, Kirkpatrick P, Gupta A et al. Effect of decompressive craniectomy on intracranial pressure and cerebrospinal compensation following traumatic brain injury. J Neurosurg. 2008;108:66-73. 
21. Ragel BT, Klimo P, Martin JE, Teff RJ, Bakken HE, Armonda RA. Wartime decompressive craniectomy: technique and lessons learned. Neurosurg Focus. 2010;28:E2.

22. Quinn TM, Taylor JJ, Magarik JA, Vought E, Kindy MS, Ellegala DB. Decompressive craniectomy: technical note. Acta Neurol Scand. 2011;123:239-44

23. Holland M, Nakaji P. Craniectomy: surgical indications and technique. Oper Tech Neurosurg. 2004;7:10-5.

24. Timofeev I, Santarius T, Kolias AG, Hutchinson PJA. Decompressive craniectomy - operative technique and perioperative care. Vienna: Springer; 2012. p. 115-36

25. Huang $X$, Wen L. Technical considerations in decompressive craniectomy in the treatment of traumatic brain injury. Int J Med Sci. 2010 7:385-90.

26. Sekhar LN, Mai JC. Dural repair after craniotomy and the use of dura substitutes and dural sealants. World Neurosurg. 2013;79:440-2.

27. Mumert ML, Altay T, Couldwell WT. Technique for decompressive craniectomy using Seprafilm as a dural substitute and anti-adhesion barrier. $\mathrm{J}$ Clin Neurosci. 2012;19:455-7.

28. Bao $Y-H$, Liang $Y-M$, Gao G-Y, Pan $Y-H$, Luo $Q-Z$, Jiang J-Y. Bilateral decompressive craniectomy for patients with malignant diffuse brain swelling after severe traumatic brain injury: a 37-case study. J Neurotrauma. 2010;27:341-7.

29. Gerl A, Tavan S. Bilateral craniectomy in the treatment of severe traumatic brain edema. Zentralbl Neurochir. 1980;41:125-38.

30. Mitchell P, Gregson BA, Vindlacheruvu RR, Mendelow AD. Surgical options in $\mathrm{ICH}$ including decompressive craniectomy. J Neurol Sci. 2007;261:89-98.
31. Senapati SB, Sekhar Mishra S, Das S, Chandra Satpathy P. Cranioplasty after decompressive craniectomy. Indian J Neurotrauma. 2012; 9:136-9.

32. Alvis-Miranda H, Castellar-Leones SM, Moscote-Salazar LR Decompressive Craniectomy and Traumatic Brain Injury: A Review. Bull Emerg Trauma. 2013;1(2):60-8.

33. Pereyra C, Benito Mori L, Schoon P, Violi D, Jacintho P, Segui G, et al. Decompressive craniectomy and brain death prevalence and mortality: 8-year retrospective review. Transplant Proc. 2012;44:2181-4.

34. Schwarz F, Dünisch P. Walter J, Sakr Y, Kalff R, Ewald C. Cranioplasty after decompressive craniectomy: is there a rationale for an initial artificial bone-substitute implant? A single-center experience after 631 procedures. J Neurosurg. 2016;124:710-5.

35. Taylor A, Butt W, Rosenfeld J, Shann F, Ditchfield M, Lewis E, et al. A randomized trial of very early decompressive craniectomy in children with traumatic brain injury and sustained intracranial hypertension. Child's Nerv Syst. 2001;17:154-62.

36. Qiu W, Guo C, Shen H, Chen K, Wen L, Huang H, et al. Effects of unilateral decompressive craniectomy on patients with unilateral acute post-traumatic brain swelling after severe traumatic brain injury. Crit Care. 2009;13:R185.

37. Cooper DJ, Rosenfeld JV, Murray L, Arabi YM, Davies AR, D'Urso P et al. Decompressive craniectomy in diffuse traumatic brain injury. $N$ Engl J Med. 2011;364:1493-502.

38. Hutchinson PJ, Kolias AG, Timofeev IS, Corteen EA, Czosnyka M, Timothy J, et al. Trial of decompressive craniectomy for traumatic intracranial hypertension. N Engl J Med. 2016;375:1119-30. 